American Journal of Infectious Diseases 4 (2): 124-130, 2008

ISSN 1553-6203

(C) 2008 Science Publications

\title{
Efficacy of Pravastatin in Non-Nucleoside Reverse Transcriptase Inhibitor (NNRTI) and Protease Inhibitor (PI)-based HAART in HIV-Infected Patients
}

\author{
Susan A. Eaton, Anita P. Rahman, Sean T. Nguyen, Amy M. Bain, Kenna D. Payne and Anthony J. Busti \\ Texas Tech University Health Sciences, Center School of Pharmacy Dallas/Fort Worth Regional Campus \\ and Dallas Veteran's Affairs Medical Center, Dallas, TX
}

\begin{abstract}
Pravastatin has generally been considered a safe and effective option for HIV-infected patients on highly active antiretroviral therapy (HAART). However, pravastatin concentrations are known to significantly decrease with concomitant efavirenz (EFV) use. Currently there are no studies determining if these reductions in pravastatin possibly translate into an attenuation of its lipid lowering efficacy when used in HIV-infected patients on non-nucleoside reverse transcriptase inhibitor (NNRTI)-based HAART. To evaluate the differences in the lipid lowering efficacy of pravastatin for the treatment of dyslipidemia in HIV-infected patients on NNRTI-based HAART compared to protease inhibitor (PI)-based regimens. A single center, retrospective evaluation of a comprehensive electronic HIV registry that identified HIV-infected, Veterans Affairs (VA) patients who received pravastatin $20 \mathrm{mg}$ plus NNRTI or PI-based HAART from January 1997 to November 2006 who met the strict criteria for inclusion. A total of 18 patients [NNRTI $(n=7)$ and PI $(n=11)$ ] met the strict criteria for inclusion. In HIV-infected patients taking NNRTI-based HAART there was a reduction in TC by $-10.1 \%$, LDL by $-12 \%$ and non-HDL by $-12.2 \%$ within 6 months after starting pravastatin 20 mg. In HIVinfected patients taking PI-based HAART, there was a reduction in TC by $-10.1 \%$, in LDL by $-21.1 \%$ and in non-HDL by $-13.8 \%$ within 6 months after starting pravastatin $20 \mathrm{mg}$. In both groups, only one additional patient achieved their patient specific lipid goals. In either group these reductions were seen without any apparent adverse drug events or compromise to virologic or immunologic control. This initial evaluation suggests that pravastatin's efficacy may be attenuated with NNRTIs versus PI-based HAART, possibly due to known reductions in pravastatin concentrations when administered with NNRTI-based regimens. These effects were seen without any apparent compromises to safety and should be validated in a prospective study.
\end{abstract}

Key words: Statins, lipids, highly active antiretroviral therapy

\section{INTRODUCTION}

Highly active antiretroviral therapy (HAART) has clearly made a significant impact on the morbidity and mortality in patients infected with the human immunodeficiency virus (HIV). [1,2] Given that HIVinfection related mortality has declined, cardiovascular disease (CVD) and the contribution of HAART to overall CVD risk, have become increasingly important. The increase in CVD as a result of HAART is supported by the Data Collection on Adverse Events of Anti-HIV Drugs Study Group (DAD), which is a large observational study consisting of approximately 23,400 patients from 11 cohorts in Europe, Australia and the United States. [3] Recently, an analysis revealed that the relative risk (RR) of myocardial infarctions per year of exposure to
HAART, adjusted for traditional CVD risk factors, was 1.16 (95\% CI; 1.09-1.23) which underscores the high degree of CVD risk in this population [3]. In an effort to address cardiovascular risk in HIV-infected patients, including lipid risk factors associated with HAART, the Infectious Diseases Society of America (IDSA) and the AIDS Clinical Trials Group (ACTG) to issue guidelines regarding the management of dyslipidemia in this highly complex patient population.

Currently, the IDSA/ACTG guidelines recommend HMG-CoA reductase inhibitors (statins) as the first line treatment option in HIV-infected patients with elevated low-density lipoproteins (LDL) and whose triglycerides (TG) are below $500 \mathrm{mg} / \mathrm{dL}$ [4,5]. Pravastatin has historically been one of a few statins of choice in this patient population due to its lower dependency of the

Corresponding Author: Anthony J. Busti, Texas Tech University Health Sciences Center School of Pharmacy Dallas Fort Worth Regional Campus 4500 S. Lancaster Rd., Bldg No. 7, Dallas, Texas 75216 Tel: 214-372-5300/240 Fax: 214-372-5020 
CYP450 isoenzyme system and thereby is theoretically less likely to result in drug interactions with various antiretroviral medications, especially protease inhibitors (PI) [6]. While most of the concerns regarding drug interaction reside with PI-based HAART, a recent pharmacokinetic study with pravastatin and efavirenz (EFV) in HIV-seronegative, adult patients revealed significant reductions in pravastatin concentrations [7]. Interestingly, there have also been reductions in pravastatin concentrations noted when given concurrently with (SQV/RTV) saquinavir/ritonavir-based regimens [8].

To date, there are no published comparative studies evaluating pravastatin's lipid lowering efficacy, safety and achievement of the National Cholesterol Education Program (NCEP) Adults Treatment Panel (ATP) III goals in HIV-infected patients being treated with nonnucleoside reverse transcriptase inhibitors (NNRTI) or PI based HAART in a real-world clinical practice setting. In addition, there is a paucity of data determining the impact that pravastatin has on the achievement of lipid goals or standards of care that clinicians are faced with in daily practice. Therefore, the primary purpose of our study was to evaluate the lipid-lowering efficacy of $20 \mathrm{mg}$ of pravastatin when used with NNRTIs and PIs as a part of HAART.

\section{MATERIALS AND METHODS}

Study design: This was a single-center, retrospective study conducted at the Veterans Affairs North Texas Health Care System (VANTHCS) and was approved by the institution review board to evaluate the lipid effects of pravastatin $20 \mathrm{mg}$ in HIV-infected patients. Patients were identified through a retrospective query of comprehensive electronic medical records, dating back from January 1997 to November 2006, within the HIV Registry and CPRS (Computerized Patient Record System) at the VANTHCS. Of note, CPRS has comprehensive patient information including access to demographics, progress notes, laboratory data, as well as inpatient and outpatient medication utilization.

Eligibility criteria: Strict, a priori defined, inclusion and exclusion criteria were used to control for known confounders that would influence the appropriate interpretation of our defined study objectives. All HIVinfected patients who had received NNRTI or PI-based HAART in conjunction with pravastatin $20 \mathrm{mg}$ were included for initial evaluation. Identified patients were then screened to determine if they met the following $a$ priori determined inclusion criteria: $\geq 18$ years of age, statin naïve for at least 3 months prior to initiation of pravastatin, no changes to any antiretroviral medications known to affect lipids pre and post initiation of pravastatin or until the first lipid level was obtained (must be within 6 months after pravastatin was initiated). Patients were also included if they were on stable doses of other lipid lowering medications (i.e., fibric acid derivatives, fish oil, ezetimibe, niacin) that had not been modified 3 months prior to initiation of pravastatin or during follow-up period.

The following exclusion criteria were used and included any changes in nucleoside reverse transcriptase inhibitor (NRTI) backbone (in particular the thymidine analogue, stavudine), uncontrolled diabetes mellitus (defined as change in HgbAlc of $\geq 1 \%$ or an average increase or decrease in serum glucose $>30-40 \mathrm{mg} / \mathrm{dL}$ ), diagnosis of evidence of nephrotic syndrome, uncontrolled or a new diagnosis of thyroid disease (TSH not within 0.4-4.5 mIU/L), evidence of significant changes in dietary and/or exercise patterns and addition or modification in the use of progestins, glucocorticoids, isotretinoin, estrogens, azole antifungals, anabolic steroids, sevelamer, red yeast rice and thiazolidinediones pre and post initiation of pravastatin.

Study objectives: The primary objective was to evaluate the lipid lowering efficacy of pravastatin $20 \mathrm{mg}$ on the lipid profile when added to a NNRTI [consisting of efavirenz (EFV) or nevirapine (NVP)] and PI-based HAART in HIV-infected patients. Pravastatin $20 \mathrm{mg}$ was chosen because this was the most common dose used at our institution in HIV-infected patients on antiretrovirals during the study period. Secondary objectives were to evaluate the effects of pravastatin when added to NNRTI and PI-based HAART on CD4 counts (cells $\mathrm{mm}^{-3}$ ), HIV viral load (copies/ $\mathrm{mL}$ ) and evaluate the attainment of NCEP ATP III LDL and non-HDL goals.

Statistical analysis: Due to the limited nature of pravastatin use in this special patient population and lack of data available in the literature, we included all available patients that met strict, a priori-defined criteria for inclusion. All statistical analyses were performed on SPSS for Windows software, version 15.0 (SPSS Inc., Chicago, IL) and median (interquartile ranges (IQR)) was reported for the majority of the parameters. A MannWhitney $\mathrm{U}$ test was used to assess continuous variables between groups and a Chi-squared test when assessing nominal data between groups. In addition, statistical analysis was also performed within groups using a 
Wilcoxin Signed Rank test to assess continuous variables and a McNemar test was used when assessing nominal data. Regardless of within group and between group comparisons, all of the analysis consisted of a comparison between baseline and post-pravastatin initiation. A twosided alpha of 0.05 was used to determine statistical significance.

\section{RESULTS}

Demographic and clinical characteristics: A total of 18 male patients [NNRTI $(n=7)$ and PI $(n=11)]$ met criteria for inclusion. Within the NNRTI group there were three patients on EFV based-HAART and four patients on NVP-based HAART. Within the PI group there were three patients on lopinavir/ritonavir, two patients on amprenavir, three patients on indinavir/ritonavir and three patients on nelfinavir-based HAART. The majority of patients were determined to be ineligible because of changes in the NRTI backbone or were on statin therapy within 3 months prior to initiation of pravastatin. The median (IQR) age of the patients in the NNRTI group was 49 (40-60) years and in the PI group it was 50 (42-55) years (Table 1). There were no significant differences in baseline characteristics between the two groups. While not statistically different, the median baseline TC, LDL and non-HDL were higher in the PI group versus the NNRTI group. In addition, there were no significant changes in body mass index (BMI), liver enzymes, thyroid function, or creatinine kinase from baseline throughout the evaluation period. None of the patients had diabetes mellitus at any point in the evaluation period.

Outcomes on lipid profiles: In the patients taking NNRTI-based HAART there was a reduction in TC by $10.1 \%$, LDL by $-12 \%$, non-HDL by $-12.2 \%$ and an increase in HDL by $+2.4 \%$ within 6 months after starting pravastatin $20 \mathrm{mg}$. Whereas in $\mathrm{HIV}$-infected patients taking PI-based HAART, there was a reduction in TC by $-10.1 \%$, LDL by $-21.1 \%$, HDL by $-4.2 \%$ and non-HDL by $-13.8 \%$ within 6 months after starting pravastatin 20 $\mathrm{mg}$. In either group these reductions were seen without any apparent adverse drug events or compromise to virologic or immunologic control.

Outcomes on goal achievement: The number of patients on NNRTI-based HAART at their patient specific NCEP III goals for LDL and non-HDL at baseline was 2/7 (28\%) and $1 / 7(14 \%)$ versus $3 / 7(43 \%)$ and $2 / 7$ (28\%) postpravastatin initiation, respectively. The number of patients on PI-based HAART at their patient specific NCEP III goals for LDL and non-HDL at baseline was $2 / 11(18 \%)$ and $1 / 11(9 \%)$ versus $3 / 11(27 \%)$ and $2 / 11$
(18\%) post-pravastatin initiation, respectively.

Safety evaluation: There were no significant differences within each group or between the NNRTI and PI groups regarding CD4 counts $(p=0.37)$ nor in the number of patients with undetectable viral loads (defined as HIV RNA viral load $<50$ copies/mL) at baseline and postpravastatin initiation. However, 1 additional patient in the NNRTI and 3 additional patients in the PI-based HAART groups achieved undetectable HIV viral loads despite already being on stable HAART.

There were no apparent adverse drug events during the evaluation period. In addition, there were no discontinuations of the pravastatin within the 6 months following initiation on stable HAART.

\section{DISCUSSION}

To our knowledge, this is the first evaluation of the comparative lipid lowering efficacy with pravastatin in HIV-infected patients on NNRTI to patients on PI-based HAART. This preliminary data suggests that the lipid lowering efficacy of pravastatin in HIV-infected patients may be attenuated when co-administered with NNRTIbased HAART (LDL reductions of $-12 \%$ in the NNRTI group versus $-21.1 \%$ in the PI group). The observed possible attenuation of LDL lowering with NNRTI-based regimen is somewhat suspected based on the results of pharmacokinetic study that evaluated the effect of atorvastatin $40 \mathrm{mg}$, simvastatin $40 \mathrm{mg}$ and pravastatin 40 mg when given with EFV in HIV seronegative patients. This study showed that the AUC of simvastatin acid was reduced by $-58 \%(\mathrm{p}=0.003)$, the AUC of atorvastatin with active metabolites was reduced by $-34 \%(p<0.001)$ and that the AUC of pravastatin was reduced by $40 \%(\mathrm{p}=0.005)$. While they did report an observed attenuation of LDL lowering with EFV with 4 days of statin use, these results were only statistically significant for the simvastatin arm nor do they reflect the effect that chronic concurrent administration used in real-world clinical practice might have on LDL-lowering efficacy [7]. The present study differs from this pharmacokinetic study in that we evaluated the lipid lowering efficacy and safety of pravastatin in both HIV-infected patients on NNRTI and PI-based HAART and after being on stable doses of the statin for at least 4 weeks to reflect a more clinically relevant scenario. Since there is a paucity of information determining the impact of lipid lowering treatment strategies in HIV-infected patients on HAART in the achievement of clinically relevant goals, we also 
Am. J. Infect. Dis., 4 (2): 124-130, 2008

Table 1: Baseline characteristics

\begin{tabular}{|c|c|c|}
\hline & NNRTI-based HAART $(\mathrm{n}=7)$ & PI-based HAART $(\mathrm{n}=11)$ \\
\hline$\overline{\text { Age (years) }}$ & $49(40-60)$ & $50(42-55)$ \\
\hline BMI $\left(\mathrm{kg} \mathrm{m}^{-2}\right)$ & $29(26-30)$ & $25(24-30)$ \\
\hline Males $n(\%)$ & $7(100)$ & $11(100)$ \\
\hline Duration of HIV (years) & $12(4-14)$ & $7(5-15)$ \\
\hline Median follow-up repeat lipid profile (days) & $59(36-109)$ & $57(30-71)$ \\
\hline CD4 count (cells mm ${ }^{-3}$ ) & $517(357-570)$ & $289(244-526)$ \\
\hline \multicolumn{3}{|l|}{ Comorbidities n(\%): } \\
\hline Hypertension & $1(14.3)$ & $3(27.3)$ \\
\hline Coronary artery disease & $2(28.6)$ & $3(27.3)$ \\
\hline Peripheral vascular disease & $1(14.3)$ & $1(14.3)$ \\
\hline Smoker & $3(42.9)$ & $5(45.5)$ \\
\hline Depression & 0 & $3(27.2)$ \\
\hline Chronic Renal Insufficiency & 0 & $1(14.3)$ \\
\hline Hepatitis $\mathrm{C}$ virus & 0 & $1(14.3)$ \\
\hline
\end{tabular}

None of the baseline characteristics between NNRTI and PI arms were statistically significant different. All data represented as median or median \pm IQR unless otherwise indicated. NNRTI $=$ non-nucleoside reverse transcriptase inhibitor, PI $=$ protease inhibitor, HAART $=$ highly active antiretroviral therapy, BMI= body mass index, TC-total cholesterol, LDL-low-density lipoprotein, TG-triglycerides, HDL-high-density lipoprotein, Non-HDL-non highdensity lipoprotein, TC/HDL - total cholesterol/high-density lipoprotein ration, TG/HDL-triglyercide/high-density lipoprotein ratio

Table 2: Median changes from baseline within NNRTI and PI-based HAART and between NNRTI and PI-based HAART

\begin{tabular}{|c|c|c|c|c|c|c|c|}
\hline & \multicolumn{3}{|c|}{ NNRTI-based HAART $(\mathrm{n}=7)$} & \multicolumn{3}{|c|}{ PI-based HAART $(\mathrm{n}=11)$} & \multirow{2}{*}{$\begin{array}{l}\text { NNRTI vs. PI-based } \\
\text { HAART Post-pravastatin } \\
\text { p-value }\end{array}$} \\
\hline & Baseline & Post-pravastatin & $\begin{array}{l}\text { Absolute } \\
\text { Difference }\end{array}$ & Baseline & Post-pravastatin & $\begin{array}{l}\text { Absolute } \\
\text { Difference }\end{array}$ & \\
\hline$\overline{\mathrm{BMI}\left(\mathrm{kg} \mathrm{m}^{-2}\right)}$ & $29.0(26-30)$ & $30.0(26-30)$ & +1.0 & $25.0(24-30)$ & $25.0(24-30)$ & 0.0 & 0.24 \\
\hline $\mathrm{TC}\left(\mathrm{mg} \mathrm{dL}^{-1}\right)$ & $227.0(215-287)$ & $204.0(185-215)$ & -23.0 & $267.0(225-317)$ & $240.0(210-266)$ & -27.0 & 0.28 \\
\hline $\operatorname{LDL}\left(\mathrm{mg} \mathrm{dL}^{-1}\right)$ & $142.0(128-154)$ & $125.0(108-133)$ & -17.0 & $166.0(117-181)$ & $131.0(105-189)$ & -35.0 & 0.85 \\
\hline $\mathrm{TG}\left(\mathrm{mg} \mathrm{dL}^{-1}\right)$ & $281.0(140-394)$ & $170.0(109-313)$ & -111.0 & $310.0(238-446)$ & $276.0(191-386)$ & -34.0 & 0.53 \\
\hline $\mathrm{HDL}\left(\mathrm{mg} \mathrm{dL}^{-1}\right)$ & $42.0(41-52)$ & $43.0(36-57)$ & +1.0 & $48.0(38-54)$ & $46.0(46-52)$ & -2.0 & 0.72 \\
\hline Non-HDL (mg dL $\left.{ }^{-1}\right)$ & $180.0(169-218)$ & $158.0(146-176)$ & -22.0 & $196.0(169-238)$ & $169.0(149-231)$ & -30.0 & 0.41 \\
\hline TC/HDL & $5.8(5.2-6.0)$ & $4.5 .0(4.3-5.5)$ & -1.3 & $6.5(4.4-7.4)$ & 5.5.0 (4.1-7.6) & -1.0 & 0.53 \\
\hline TG/HDL & $6.7(3.4-7.6)$ & $4.7 .0(2-6.8)$ & -2.0 & $7.7(3.8-10.2)$ & $5.8 .0(4.2-10)$ & -1.9 & 0.47 \\
\hline CD4 count (cells mm ${ }^{-3}$ ) & $517.0(357-570)$ & $429.0(182-719)$ & -88.0 & $289.0(244-526)$ & $337.0(227-542)$ & +48.0 & 0.37 \\
\hline HIV viral load & & & & & & & \\
\hline$\leq 50$ copies $\mathrm{mL}^{-1}$ & $57.0 \%$ & $71.0 \%$ & $+14.0 \%$ & $36.0 \%$ & $64.0 \%$ & $+28.0 \%$ & $\mathrm{Ns}^{\mathrm{a}}$ \\
\hline
\end{tabular}

All data represented as median or median \pm IQR unless otherwise indicated. ${ }^{a}=$ Not significant,NNRTI $=$ non-nucleoside reverse transcriptase inhibitors, PI $=$ protease inhibitors, HAART $=$ highly active antiretroviral therapy, BMI = body mass index, TC-total cholesterol, LDL-low-density lipoprotein, TG-triglycerides, HDL-high-density lipoprotein, Non HDL-non high-density lipoprotein, TC/HDL - total cholesterol/high-density lipoprotein ration, TG/HDL-triglyercide/high-density lipoprotein ratio

evaluated how this intervention translated into the achievement of the same standards of care as those applied to HIV-negative patients (i.e., NCEP ATP III goals).

While our results show a relatively blunted LDLlowering efficacy when pravastatin is used concurrently with NNRTIs, the exact mechanism by which this attenuation in efficacy might occur needs further evaluation. The attenuated lipid response is not unexpected and most likely related to a reduction in pravastatin concentrations similar to the observations of Gerber and colleagues [7]. The mechanism by which the pravastatin concentrations may decrease with coadministration of EFV has yet to be fully elucidated. It has been shown that both NNRTIs are able to induce the activity of efflux pump P-gylcoprotein, for which pravastatin is a known substrate [9]. In addition, EFV could potentially upregulate activity of multi-specific organic anion transporter (cMOAT/MRP2) given that these two transport mechanisms are under common genetic regulation $[10,11]$. Both of these actions could possibly explain he blunted lipid-lowering response observed in our study. While there are some pharmacokinetic and pharmacodynamic data available with statins and EFV, there is little known about the other commonly prescribed NNRTI, NVP.

Similar to EFV, NVP is also a known inducer of the CYP450 3A4 isoenzyme system [12,13,14]. Unfortunately, there are no pharmacokinetic and/or pharmacodynamic data with coadministration of NVP and statins. As a result it is not known if there is a similar attenuation of lipid lowering efficacy with coadministration of NVP and pravastatin. Interestingly, a subgroup analysis within the NNRTI group in our study, 
suggests that NVP may possibly be attenuating the LDL lowering efficacy to a greater extent than those on EFVbased HAART $(-3.7 \%$ versus $-21.7 \%$, respectively). This may be due to the known greater inductive properties of NVP on P-gp than EFV [9]. However, due to the small sample size of the EFV and NVP sub-groups, this can only be viewed as a hypothesis generating observation since no other data exists at this time. While there are data with EFV and statins showing reductions in pravastatin concentrations, there is also data with PIs that have shown conflicting results (some suggesting similar reductions as seen with EFV and some showing increases in pravastatin concentrations) $[8,15]$. In particular, a pharmacokinetic study where SQV/RTV, a known potent inhibitor of CYP 3A4, was given to HIV seronegative patients resulted in an exponential increase in the area under the curve (AUC) for simvastatin $40 \mathrm{mg}(3059 \%, \mathrm{p}<0.001)$ and total active atorvastatin AUC from $40 \mathrm{mg}(79 \% \mathrm{p}<0.001)$. However, this same study also reported that SQV/RTV caused a decrease in pravastatin's (40 mg) AUC by $50 \%$ $(\mathrm{P}=0.005)$ [8]. While the significant elevations in the simvastatin and atorvastatin levels are explained by their dependency as substrates for the CYP3A4 isoenzyme, the mechanism for the reductions in pravastatin concentrations are not currently known. It has been speculated that since pravastatin is metabolized via glucuronidation and sulfation, the inducing properties of RTV on the glucuronidation pathway may be potentially contributing to this drug interaction [16]. Unfortunately, this pharmacokinetic study did not report the lipid lowering effects of any of the statins studies, thus it could not be determined if there was an attenuation of the LDL lowering with pravastatin. None of the patients in our study were on RTV/SQV-based HAART. In addition, $\mathrm{RTV} / \mathrm{SQV}$ is generally no longer used in clinical practice and thus may not be as useful today. As a result we evaluated the lipid lowering efficacy of pravastatin in PI's in general to determine if there were any observed attenuations as seen with NNRTIs.

In HIV-infected patients from our study on PI-based HAART who received pravastatin $20 \mathrm{mg}$ there was a reduction in LDL of $-21 \%$, which as stated above was a greater reduction than that seen in NNRTI-based HAART. Taking our observations and comparing them to the known efficacy documented in the primary literature for HIV-negative patients can be of further value. An evaluation of available literature would show that the LDL reductions seen with pravastatin $20 \mathrm{mg}$ in HIV-negative patients ranges from -24 to $-32 \%$ [17,18]. These observations in HIV-negative patients from the literature would further suggest that LDL lowering efficacy in HIV- infected patients on either NNRTI or PI-based HAART maybe slightly attenuated when using the same dose of pravastatin. This would require a prospective evaluation to confirm. In the meantime, our observations may to be useful for clinicians to consider especially in those difficult to treat patients. Additionally, what may be more important for practicing clinicians faced with treating this patient population is the ability of pravastatin $20 \mathrm{mg}$ to be safely used and to help patients achieve their specific NCEP ATP III goals.

When evaluating the achievement of patient specific NCEP ATP III lipid goals, one additional patient in each of the study groups were able to achieve both LDL and non-HDL goals. The low achievements of NCEP ATP III goals in both groups could be due to a number of factors that are important to note; 1) the lower doses of pravastatin used may not have allowed for greater reductions in lipids needed; 2) the known and established difficulty in treating HIV-infected patients with antiretroviral associated dyslipidemia and; 3 ) the possible lack of similar lipid lowering efficacy of pravastatin when used with antiretrovirals. Lastly, it appears that the CD4 count and viral load remained stable, signifying that pravastatin did not affect the virologic and immunologic efficacy of either the NNRTI or PI-based HAART.

As with any retrospective study and despite the implementation of a-priori defined strict criteria for inclusion, there are several limitations to our study. Present study included a small sample size due to our strict criteria for inclusion used to control for known confounders of our endpoints. This limitation is also unfortunately a limitation in much of the data evaluating lipid lowering treatment strategies in HIV-infected patients with dyslipidemia being treated with HAART. The evaluation of pravastatin $20 \mathrm{mg}$ was chosen as this was the most common dose used in our institution for HIV-infected patients on HAART. While it is not specifically known, the use of lower doses of pravastatin in our institution may reflect clinician's hesitancy to use larger doses or to appropriately titrate doses due to perceived risk for increases in adverse drug reactions in this patient population. Also, being that our study site was based at a VA medical center, our study population consisted of all males and thus our observations may not be similar in female HIV-infected patients on HAART. Lastly, due to the fact that this study was retrospective, fasting lipid levels could not be absolutely assured. However, it is a common practice at our institution for our patients to consistently come in for labs in a fasting state to avoid potential confusion.

Given the degree of complexity when trying to treat 
this patient population to the same standards of care for non-HIV patients, this retrospective data still provides needed and relevant observations that may be useful to clinicians caring for these special patients with complex regimens. As a result of the increase incidences in CVD, potential number of drug interactions and complex antiretroviral regimens, this patient population is in need of multiple treatment strategies in order to effectively treat for dyslipidemia while also maintaining viral and immunological control.

\section{CONCLUSION}

Our findings suggest that pravastatin's lipid lowering efficacy may be attenuated in both NNRTI and PI based HAART when compared to established efficacy parameters in non-HIV patients seen in the current literature. In addition, it is possible that the greatest attenuation of pravastatin's efficacy may be seen with NNRTI based regimens. The exact mechanism of the drug interaction seen in pravastatin and NNRTI and PIs is currently speculative and requires additional studies; however, knowing that the efficacy of pravastatin $20 \mathrm{mg}$ could be attenuated with HIV-infected patients on HAART would highlight the need for higher doses of pravastatin to reduce lipid levels and achieve NCEP ATP III lipid goals in this difficult to treat, special patient population. Our results should serve as proof of concept data that should be validated prospectively.

\section{REFERENCES}

1. Mocroft, A., R. Brettle, O. Kirk, A. Blaxhult, J.M. Parkin, F. Antunes, P. Francioli, A. D'Arminio Monforte, Z. Fox and J.D. Lungren, 2002. Changes in the cause of death among HIV positive subjects across Europe: Results from EuroSIDA study. AIDS, 16: 1663-1671.

2. Palella, F.J., K.M. Delaney, A.C. Moorman, M.O. Loveless, J. Fuhrer, G.A. Satten, D.J. Aschman and S.D. Holmberg, 1998. Declining morbidity and mortality among patients with advanced human immunodeficiency virus infection. N. Engl. J. Med., 338: 853-860.

3. DAD Study Group, 2007. Class of antiretroviral drugs and the risk of myocardial infarction. N. Engl. J. Med., 356: 1723-1735.

4. Expert Panel on Detection Evaluation and Treatment of High Blood Cholesterol in Adults, 2001. Executive summary of the third report of the National Cholesterol Education Program (NCEP) Expert Panel on Detection, Evaluation and Treatment of High Blood Cholesterol in Adults (Adult Treatment Panel III). JAMA, 285: 2486-2497.
5. Dube, M.P., J.H. Stein, J.A. Aberg, C.J. Fitchenbaum, J.G. Gerber, K.T. Tashima, K.W. Henry, J.S. Currier, D. Sprecher and M.J. Glesby, 2003. Guidelines for the evaluation and management of dyslipidemia in human immunodeficiency virus (HIV)-infected adults receiving antiretroviral therapy: Recommendations of the HIV medicine association of the Infectious Disease Society of America and the Adult Aids Clinical Trials Group. Clin. Infect. Dis., 37: 613-627.

6. Lennernas, H. and G. Fager, 1997. Pharmacodynamic and pharmacokinetics of the HMG-CoA reductase inhibitors. Similarities and differences. Clin. Pharmacokinet., 32: 403-425.

7. Gerber, J.G., S.L. Rosenkranz, C.J. Fichtenbaum, J.M. Vega, A. Yang, B.L. Alston, S.W. Brobst, Y. Segal and J.A. Aber, 2005. Effect of efavirenz on the pharmacokinetics of simvastatin, atorvastatin and pravastatin. J. Acquir. Immune. Defic. Syndr., 39-307-312.

8. Fichentenbaum, C.J., J.G. Gerber, S.L. Rosenkranz, Y. Segal, J.A. Aber, T. Blaschke, B. Alston, F. Fang, B. Kosel and F. Aweeka, 2002. Pharmacokinetic interactions between protease inhibitors and statins in HIV sero-negative volunteers: ACTG Study A5047. AIDS, 16: 569-577.

9. Neuvonen, P.J., N. Mikko and J.T. Backman, 2006. Drug interactions with lipid-lowering drugs: Mechanisms and clinical relevance. Clin. Pharmacol. Ther., 80: 565-581.

10. Stormer, E., L.L. von Moltke, M.D. Perloff and D.J. Greenblatt, 2002. Differential modulation of P-glycoprotein expression and activity by nonnucleoside HIV-1 reverse transcriptase inhibitors in cell culture. Pharm. Res., 19: 1038-1045.

11. Chandra, P. and K.L. Brouwer, 2004. The complexities of hepatic drug transport: Current knowledge and emerging concepts. Pharm. Res., 21: 719-735.

12. Erickson, D.A., G. Mather, W.F. Trager, R.H. Levy and J.J. Keirns, 1999. Characterization of the in vitro biotransformation of the HIV-1 reverse transcriptase inhibitor nevirapine by human hepatic cytochromes P-450. Drug. Metab. Dispos., 27: 1488-1495.

13. Piscitelli, S.C. and K.D. Gallicano, 2001. Interactions among drugs for HIV and opportunistic infections. N. Engl. J. Med., 344: 984-996.

14. Riska, P., M. Lamson, T. MacGregor, J. Sabo, S. Hattox, J. Pav and J. Keirns, 1999. Disoposition and biotransformation of the antiretroviral drug nevirapine in humans. Drug. Metab. Dispos., 8: 895-901. 
15. Panel on Antiretroviral Guidelines for Adult and Adolescents. Guidelines for the use of antiretroviral agents in HIV-infected adults and adolescents. Department of Health and Human Services. October 10, 2006; 1-113. Available at http://www.aidsinfo.nih. gov/ContentFiles/AdultandAdolescentsGL.pdf. Accessed (July 2, 2007).

16. Ouellet, D., A. Hsu, J. Qian, C.S. Locke, C.J. Eason, J.H. Cavanaugh, J.M. Leonard and G.R. Granneman, 1998. Effect of ritonavir on the pharmacokinetics of ethinyl oestradiol in healthy female volunteers. Br. J. Clin. Pharmacol., 2: 111-116.
17. Pravastatin (Pravachol ${ }^{\circledR}$ ) Product insert. BristolMyers Squibb, Inc., Princeton, NJ. April 2007.

18. Jones, P.H., M.H. Davidson, E.A. Stein, H.E. Bays, J.M. Mckenney, E. Miller, V.A. Cain and J.W. Biasetto, 2003. Comparison of the efficacy and safety of rosuvstatin versus atorvastatin, simvastatin and pravastatin across doses (STELLAR Trial). Am. J. Card., 93: 152-160. 